\title{
Perceiving the centroid of configurations on a rolling wheel
}

\author{
DENNIS R. PROFFITT and JAMES E. CUTTING \\ Wesleyan University, Middletown, Connecticut 06457
}

\begin{abstract}
Undergraduates observed configurations of point-lights undergoing wheel-generated motions and judged how wheel-like the movement of each stimulus appeared on a 7-point scale. Viewer judgments were predicted by a metric defining the variable parameters for the motion path of each configuration's geometric center-the centroid. The effects on judgments of eye movement and the stimulus characteristics of rotation, translation, and configuration were explored in six experiments. First, a strain operation on the dynamic stimuli did not affect the ability of the metric to predict perceptual judgments. Second, the predictive strength of the metric did not interact with the type of eye movements used in viewing the stimuli, though judged wheel-likeness was greater under pursuit vision than under static fixation. Third, variations in the extent of translation yielded little, if any, effect on observers' judgments, nor did translation in a circular path. Finally, for stimuli having two lights extremely close together in the configuration, the metric's predictive value was slightly lessened but only at the limits of visual acuity. Thus, within a wide range of presentation conditions, and for a wide variety of configurations, a metric that defined the variable parameters for the motion path of the centroid was an accurate predictor of observers' judgments of goodness of perceived rotary motion.
\end{abstract}

For over a quarter of a century, two voices, one in American and one in Europe, have urged perceptual psychologists to study the perception of events in which the information available to observers is manifested over time. Gibson $(1950,1966,1979)$ and Johansson $(1950,1973,1975)$ have remained unconvinced that studying the perception of static forms can yield insights into dynamic event perception. Rather, they have argued that static perception is a special case of event perception.

The first steps in investigating event perception have addressed the problem of specifying what is perceived. As Turvey $(1977$, p. 82$)$ remarked, "In a phrase, our conception of the what of processing determines our investigation and interpretation of the how of processing." What is sought is a description both of the observer's experience and of the information present in the event that supports it. For any event, such a description will be one mathematical specification of the event's invariant structure. Since

This research was supported by small grants to both authors from Wesleyan University and made possible by the Department of Psychology of Stanford University, where the second author spent a sabbatical leave. We thank Jacob Beck for suggesting Experiment 2, Roger Shepard for suggesting Experiment 6, and Peter Smith for technical assistance. We also thank Wistar Comfort, of the Wesleyan University Department of Mathematics, for his assistance. Reprint requests should be sent to Dennis R. Proffitt, Department of Psychology, Gilmer Hall, University of Virginia, Charlottesville, Virginia 22901, or to James E. Cutting, Department of Psychology, Wesleyan University, Middletown, Connecticut 06457. any complex event may have an indefinite number of possible mathematical descriptions, our search is for the particular description selected by the perceptual system.

In previous research (Proffitt, Cutting \& Stier, 1979), as well as in the experiments reported here, we have examined the perception of wheel-generated motions. This phenomenon has a long history from mathematics and philosophy through Gestalt psychology. It has drawn attention because the perceived motions of a rolling wheel are not obviously manifested in the motions of the wheel's individual parts. When a wheel is rolled along a level path, every point on its perimeter traces a path called a cycloid, as shown in the top panel of Figure 1, where the center of a rolling wheel is represented as a small dot and a perimeter point as a large dot. Every point within the perimeter of the wheel, except the center, traces a curve called a prolate cycloid which is a smoother curve with a smaller vertical excursion than a cycloid. One such curve is drawn in the middle panel of Figure 1.

Rubin (1927) first brought to the attention of psychologists the fact that one does not see the cycloidal motion of a point on the perimeter of a rolling wheel. Instead, one sees the perimeter points revolving about the wheel's center and the center moving with a linear translatory motion. The bottom panel of Figure 1 represents this perception. Rubin argued from this phenomenon for the oft-repeated Gestalt dictum: One does not perceive the motion of 


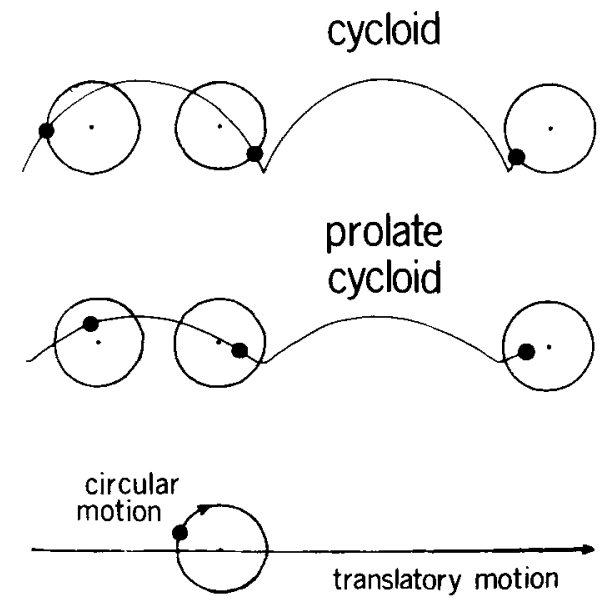

Figure 1. In the top panel is shown the motion of two points on a rolling wheel, with the center represented as a small dot and a perimeter point as a large dot. The middle panel likewise presents the motion of two points on a rolling wheel, one being the center and the other a point within the perimeter. The lower panel shows the circular and translatory components of motion.

a uniform whole by perceiving individually the movement of its various parts.

We have studied wheel-generated motions by creating stimuli similar to those used first by Duncker $(1929 / 1937)$. One to four point-light sources were placed on a wheel which was video-taped as it rolled on a level track in a dark room. Figure 2 shows two examples. In the top panel is a wheel with lights attached to its perimeter $120^{\circ}$ apart: These three point-lights are seen to revolve about the wheel's invisible center, and the whole system of lights is seen to move linearly. The bottom panel of Figure 2 shows a wheel with three lights attached, two on the perimeter and one within. This stimulus appears to move in a quite different manner: The three pointlights revolve about a center within the triangle that they define, and the system of lights hop along a cycloidal path traced by this center.

We determined that the perceived center of rotation for any system of lights on a rolling wheel corresponds to the centroid of the geometric form defined by taking the point-lights as vertices. The internal dynamics, the motion of the lights relative to each other, is perceived as a rotation about this center of its gemetric form. Angular displacement, the movement of the whole system of lights relative to the observer, is perceived as the motion of the system's centroid.

Assuming that the point-lights define an area within any arbitrarily placed coordinate system, the centroid can be determined by the following definite integrals:

$$
\bar{x}=\frac{\int_{a}^{b} x h(x) d x}{\int_{a}^{b} h(x) d x}, \quad \bar{y}=\frac{\int_{c}^{d} y l(y) d y}{\int_{c}^{d} l(y) d y},
$$

where $\bar{x}$ and $\bar{y}$ are the coordinates for the centroid, $h(x)$ and $l(y)$ are the lengths of the bounded slices of the area taken vertically and horizontally, respectively, and $a$ and $b$ are the extreme values of $x$, and $c$ and $d$ are the extreme values of $y$. The numerator of the first equation is called the first moment of the area with respect to the $y$-axis, and the numerator of the second is called the first moment of the area with respect to the $\mathrm{x}$-axis. The centroid of a plane area is called the center of gravity (or center of mass) of that area. In physics, the displacement of a rotating or vibrating object is determined by regarding the center of mass as a single particle subject to the applied external force; thus, the motion of the center of mass is called the translational motion of the object. Finally, if the area is symmetric with respect to any line in its plane, its centroid lies on this line; moreover, if the area has a center of symmetry, this point is the centroid.

The centroid for any system of lights will hop along a cycloidal path for all stimuli except those in which the centroid corresponds to the center of the wheel. In this latter case, of course, the movement of the centroid will be linear. The vertical excursion of the movement of the centroid is twice the distance of the centroid from the wheel's center. Figure 3 shows a wheel with three lights attached. The distance from the centroid to the wheel's center we call $D_{m}$. Thus, the vertical excursion of the centroid is $2 \mathrm{D}_{\mathrm{m}}$ every $2 \pi \mathrm{r}$ of horizontal translation. The rotational component of motion defines a circular path of radius equal to $\left(r-D_{m}\right)$.

Each stimulus was assigned a metric, $D_{m} / r$, the distance of the centroid from the wheel's center expressed as a proportion of the wheel's radius. This metric accounts for the variable parameters of cycloidal
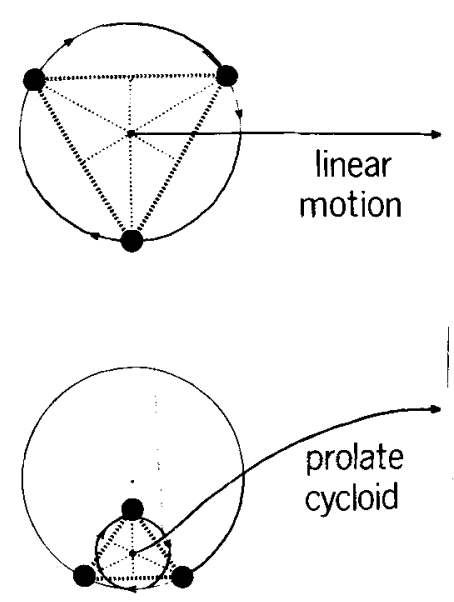

Figure 2. The top panel shows the percejved motion components for $a$ wheel which has lights attachei to the perimeter $120^{\circ}$ apart. The bottom panel shows the perceived mution components for a wheel with three lights attached, two on the perimeter and one within. 


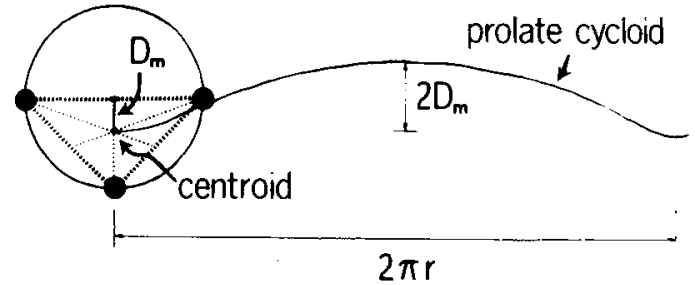

Figure 3. The perceived cycloidal motion of a three-light configuration on a rolling wheel is shown. $D_{m}=$ the distance from the centroid to the center of the wheel, $r=$ the radius of the wheel.

motion for any system of lights on a rolling wheel. As the metric's value approaches zero, the centroid approaches the center of the wheel: therefore, the greater $D_{m} / r$, the more the stimulus appears to hop.

When observers made judgments of the wheel-like character of movement on a 7-point unipolar scale, with 7 indicating the most and 1 the least wheellike movement, $D_{m} / r$ accounted for a mean of $85 \%$ of all the variance in goodness judgments across five studies employing 27 different stimulus configurations. Moreover, we found little or no support for three notions about rotary motion found in the previous literature. First, Koffka (1935) stated that the goodness of a rotating figure would, in part, depend on the number of lights mounted on the wheel. Second, by inference from the demonstrations of Duncker (1929/1937) and Johansson (1973), we can assume that they believed the center of the wheel was a special location, which, if marked by a light, would promote the perception of wheel-like form more than other points so marked. Finally, Verbrugge and Shaw (Note 1) suggested that the rotational symmetry of the lights is important to perceptual goodness of wheel-like motion. We found, however, that configurations mattered much more than (a) the number of lights, (b) their individual location with respect to the generating wheel, or (c) their rotational symmetry. Moreover, the important configurational property was the relation of the abstract (unseen) centroid of the light system to the abstract (unseen) center of the generating wheel. This relation is manifested only over time and is not discernible from a static presentation.

Previous statements about the perception of rotary motion divide into three camps. All discussants of this event note that rotary motion divides into two components, rotation and translation. That is, we see an object rotating and we also see it moving across the visual field. Two views, however, go beyond this observation by postulating the order in which these two motion components are extracted from the events. Wallach (1965) notes that we first extract information defining the internal dynamics of the lights (which he calls object-relative motion), and then the residual motion is perceived as the translation across the visual field (which he calls angular displacement). Johansson (1973), on the other hand, states that we extract the common motion vector first-linear translation is a common component vector of all points undergoing wheel-generated motion-and after extracting this information perceive the systemic rotation, which is the residual. In other words, by Wallach's account, we extract rotation then translation, and by Johansson's account, we extract translation then rotation. Our previous study found strong support for Wallach's view (Proffitt et al., 1979).

In the present series of studies, we looked at more specific aspects of the perception of rotary motion: In the first study, we examined the perceptual importance of rigid rotation; in the second, the role of eye movements in perceiving the translatory component of rotary motion; in the third, fourth, and fifth, aspects of translation; and in the sixth and final study, proximity constraints of pairs of lights in the overall configuration.

\section{GENERAL METHOD}

Dynamic stimuli for all experiments were generated on a Data General Nova computer with similar FORTRAN programs, and displayed on a Tektronix 604 monitor. Wheel-like configurations appeared as if attached to a wheel that rolled across the screen in 3 revolutions at $1 \mathrm{rev} / \mathrm{sec}$, traversing a visual angle of about $15^{\circ}$ for all viewers. Stimuli simulated two or three lights mounted on a wheel whose rim was not visible. See, for example, the left panel of Figure 4. Each light subtended a visual angle of about $5 \mathrm{~min}$ of arc; measured vertically, stimuli subtended a maximum visual angle of $1^{\circ}$ to $3^{\circ}$, depending on configuration and experimental condition.

The six basic stimuli used in Experiments 1 through 5 consisted of three pairs of items (one stimulus with three lights and the other with two), each pair differing from the other two in their centroid's distance from the center of the wheel that generated the movement. This distance, expressed as a proportion of the wheel's radius, is $D_{m} / r$. Two stimuli had $D_{m} / r$ indices of .00 , two had indices of .34 , and two of .75 . All six had been used previously by Proffitt et al. (1979). The 10 stimuli of Experiment 6 were variations on this theme.

Twenty-eight Stanford University undergraduate students participated in the studies. They received either introductory psychology course credit or $\$ 2.50$ for their efforts. Each participated in two or three of the six studies presented here, in groups of one to four. The order in which they viewed the test orders (one per experiment) was not counterbalanced; our previous studies have shown results to be so stable that context and prior experience appear to produce minimal effects. For all experiments, viewers were instructed to use a 7 -point unipolar scale, with 7 indicating the most and 1 the least wheel-like movement. Our use of this scale was based on the intuition that observers would judge one stimulus as more wheellike if it appeared to hop less than another.

In Experiments 1, 2, 3, and 6, each stimulus was presented three times in consecutive random orders, and in Experiments 4 and 5 , each was presented six times. In all experiments, the first judgment of each item was discarded, serving as practice in order to stabilize the use of the judgment scale. Each trial was preceded by a warning light presented in the middle of the screen, with $12 \mathrm{sec}$ between onsets of trials.

\section{EXPERIMENT 1: RIGID AND NONRIGID ROTATION}

Johansson (1974a) and Shaw and Pittenger (1977) have argued that invariances in the optical flow 
are often more abstract than Euclidean metrics can describe. Shaw and Pittenger discussed the Euclidean property of rigidity as follows: "By a rigid object (or shape) we mean one such that the distance between any pair of points on its surface remains unchanged when the object is transformed by displacement or reflection.... The full set (or group) of transformations which constitute symmetry operations for rigid objects (that is, leaves their shape unchanged) is exactly what we mean by Euclidean geometry. Rotation, translation, and reflection are the transformational invariants of Euclidean space" (p. 113).

In our previous studies of wheel-generated motions (Proffitt et al., 1979), the rigidity of the point-lights, and thus the symmetry of Euclidean space, had been preserved in all cases. We sought to confirm the applicability of non-Euclidean metrics in this study by breaking the rigid symmetry of our stimuli. This was done via a strain operation that vertically foreshortened the wheel shape. The geometry that results from a strain operation is called affine geometry.

Three strain operations were employed, causing the rotation component of movement for the pointlights to describe ellipses of varying degrees of flatness. As any system of lights travels about an ellipse, the distance between pairs is constantly changing. $\mathrm{D}_{\mathrm{m}} / \mathrm{r}$, however, remains invariant. The rotational path of the centroid for any light system is an ellipse with eccentricity equal to the generating ellipse.
Thus, the distance of the centroid to the center of the ellipse relative to the length of the line from the ellipse's center, through the centroid to the perimeter, is constant. Our question was whether these three types of systemic movements would appear equally wheel-like.

\section{Method}

Stimuli 1 through 6, shown in the left panel of Figure 4, were presented in three different moving configurations, where the $y$-axis excursion of each light was $1.0, .50$, or .25 of that of the $x$-axis excursion. All 18 stimulus configurations were randomized within one test sequence. Ten viewers participated in this study. Although they were not explicitly informed to interpret the stimuli as wheels viewed from different orientations, they were asked to rate the movements as more or less wheel-like on a 7-point scale.

\section{Results and Discussion}

As shown in the right panel of Figure 4, the distance measure $\left(D_{m} / r\right)$ accounted for most of the variance in the results $[F(2,18)=37.0, p<.001]$. That is, judgments of the 18 stimuli were much more strongly correlated $(r=-.93, p<.001)$ with $D_{m} / r$ than with any other variable. This result is virtually identical to that of Proffitt et al. (1979). Of particular interest, there was no effect of foreshortening in the vertical axis $[\mathrm{F}(2,18)=1.17$, ns; $\alpha=.05]$. Moreover, as in our previous studies, there was only a marginal effect of the number of lights in the stimulus $[F(1,9)=4.29, p<.07]$, with all threelight systems appearing slightly more wheel-like than

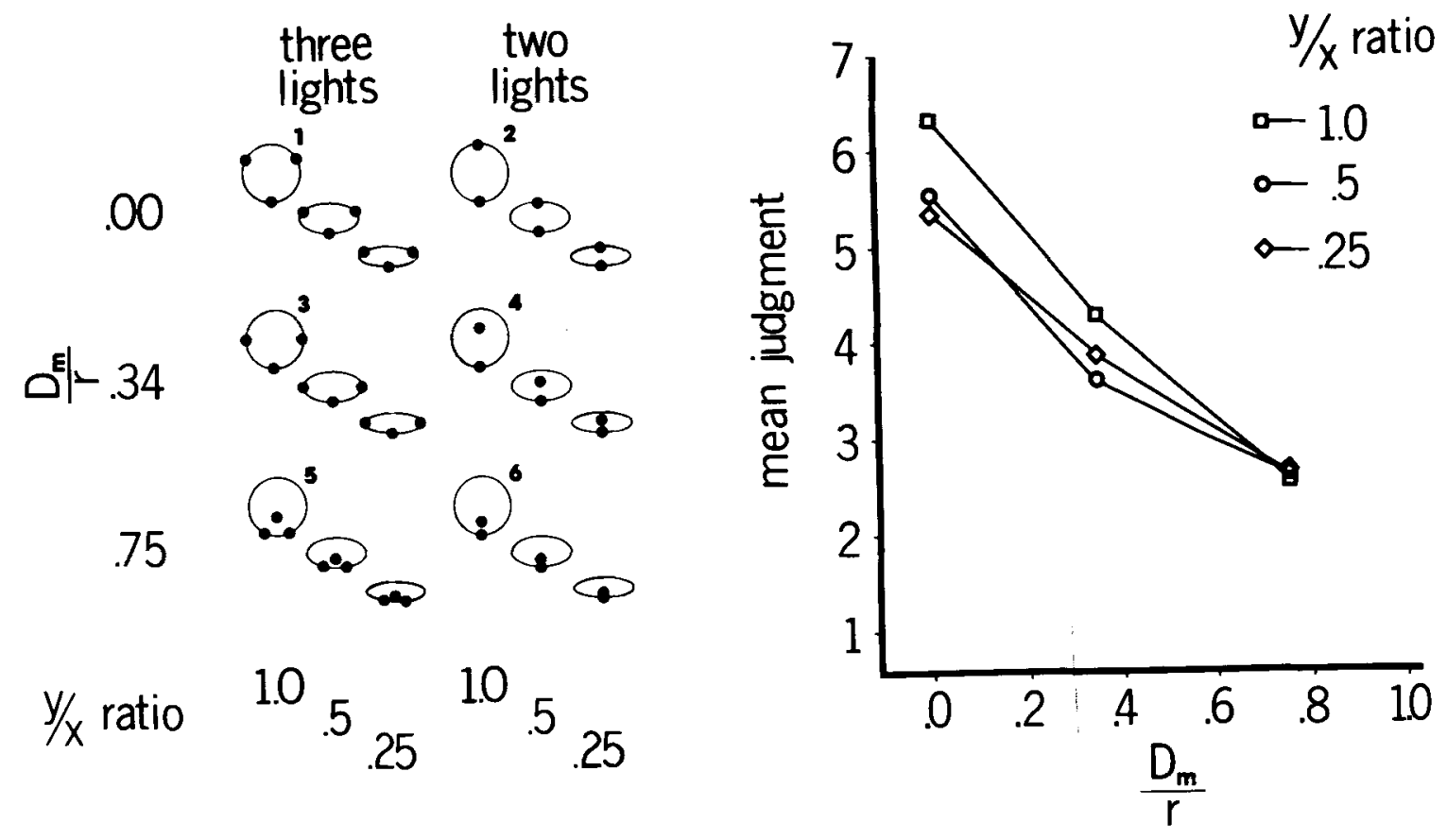

Figure 4. In the left panel are shown the six stimuli (numbered 1 through 6 ) used in the first five experiments. In Experiment 1, those six were presented in three configurations of relative foreshortening. In the right panel, collapsing across stimuli with two and three lights, the results are shown as a function of judgments, $y / x$ ratio (relative vertical foreshortening), and $D_{m} / r$ (the distance from the midpoint of the light system for each stimulus to the generating center of the movement, as a proportion of the radius). 
two-light systems. Standard errors of the mean for each stimulus configuration in this study, as well as in all others presented here, consistently average about $1 / 3$ of a judgment point.

Thus, in answer to our experimental question, the Euclidean property of rigidity in a configuration of lights is not necessary for the perception of the motion invariant described by $D_{m} / r$. The centroid of the system of lights remains a potent factor in perception. Despite the ellipsoidal structure of two-thirds of the stimuli, the centroid always remains a fixed-ratio distance from the center. The systematic correlation between $\mathrm{D}_{\mathrm{m}} / \mathrm{r}$ and judgments suggests that an object's motions are perceived from dynamic relations among its parts and not from particular patterns of movements of individual parts. The three conditions of foreshortening, which yield different movement vectors, do not yield the perception of different motions.

\section{EXPERIMENT 2: EYE MOVEMENTS AND HORIZONTAL TRANSLATION}

If perception of rotary motion is a function of the difference between the centroid of the configuration of lights and the generating center of the movement, as suggested by the results of Proffitt et al. (1979) and the results of Experiment 1, how is that perception mediated? In other words, is the information picked up more or less directly, as Gibson (1966, 1979) would suggest, or is it done through some other means, perhaps through the self-monitoring of eye movements? It may be that the centroid of the group of lights is followed by pursuit eye movements as it describes a series of prolate cycloids. This pattern of movements may then be compared against the

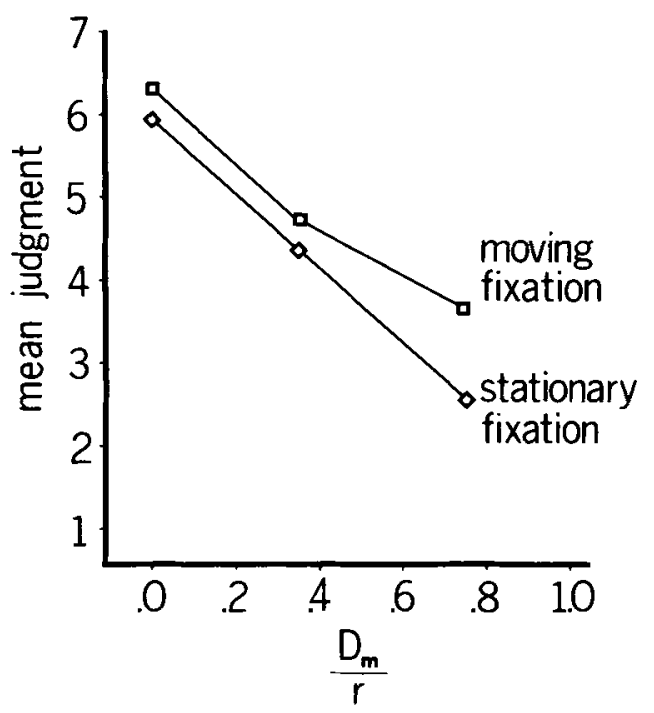

Figure 5. Results of Experiment 2, collapsing across stimuli with two and three lights. The moving-fixation condition entailed pursuit viewing of the rolling systems of lights, whereas the stationaryfixation condition entailed nonpursuit viewing. knowledge that following the center of a true wheel requires no cycloidal pattern, but merely a flat translation across the viewing field. Such a result would be consistent with the views of Stern and Emelity (1978) and Stoper (1973). In the current study, two experimental conditions were designed to test this possibility.

\section{Method}

The six stimuli, numbered 1 through 6 in the left panel of Figure 4 , were presented in two fixation conditions. In one condition, viewers focused on a stationary fixation point at midscreen, $2^{\circ}$ above the center of the path of the wheel-like system of lights, which rolled underneath. In the other, they focused on a laterally moving point always $2^{\circ}$ above and $2^{\circ}$ in front of the unseen center of the wheel that generated the moving system. This fixation point did not describe a cycloid, or prolate cycloid; instead, it moved linearly with the speed of the stimulus. Ten viewers participated in the study, rating the wheel-likeness of the stimuli. The two conditions were randomized within one test sequence, and the type of trial was announced by a warning light that either remained fixed at midscreen (indicating a stationary fixation trial) or moved linearly across the screen (indicating a moving fixation trial).

\section{Results and Discussion}

As shown in Figure 5, the distance measure was again a strong predictor of judgments $[F(2,18)=$ $19.7, \mathrm{p}<.001]$. Fixation condition was also potent $[F(1,9)=36.0, p<.001]$, with stimuli viewed under moving-fixation conditions judged as considerably more wheel-like than the same stimuli under stationaryfixation conditions. This second result is consistent with that of Stern and Emelity (1978) and Stoper (1973) on the contribution of eye movements to dynamic form perception. There was no reliable interaction between the distance index and fixation condition $[F(2,18)=3.66$, ns: $\alpha=.05]$.

Despite the main effect of fixation condition, we consider the major contribution of this experiment to be the continued demonstration of the correlation of $\mathrm{D}_{\mathrm{m}} / \mathrm{r}$ with judgments $(\mathrm{r}=-.93, \mathrm{p}<.001)$, irrespective of eye movements. Although we were not able to monitor eye movements directly, we take the fixation effect as evidence that viewers followed instructions. Comparing these results with those of Experiment 1 and of Proffitt et al. (1979), rotary judgments appear to be remarkably independent of what the eyes are doing. Thus, perception of rotary motion as related to our distance measure cannot be mediated solely through eye movements. This outcome is consistent with the view that $D_{m} / r$ is a stimulus invariant picked up by the visual system directly, and orthogonal to concerns of Stern and Emelity (1978).

\section{EXPERIMENT 3: VARIED HORIZONTAL TRANSLATION}

The original formulation of $D_{m} / r$ (Proffitt et al., 1979) was ambiguous in that it did not explicitly state what $r$ was the radius of. Two types of radii 
can be differentiated. One is the radius of the circular surface on which the light configuration rolls, the axle radius. The other is that of the outermost light (or lights) with respect to the center of the generating wheel, the system radius. Our previous studies (Proffitt et al., 1979, and the present Experiments 1 and 2) confounded these two measures completely: All stimuli had at least one light mounted on the perimeter of the wheel generating the movement, and had no light mounted beyond that perimeter. If lights are mounted beyond the perimeter of the generating wheel, they would describe not a series of cycloids or prolate cycloids, but, instead, a series of curtate cycloids (see Johansson, 1974b). Une such curve is drawn in the top panel of Figure 6. The vertical excursion of a curtate cycloid is twice the system radius, whereas the horizontal translation is $2 \pi$ times the axle radius each rotational period.

An examination of the bottom panel of Figure 6 shows that $D_{m} / r$ will express two quite different relations, depending on what $r$ is taken to be the radius of. If it is taken as the axle radius, then $D_{m} / r$ is the ratio of the vertical motion of the centroid $\left(2 D_{m}\right)$ to the translational distance traveled each period $\left(2 \pi \mathrm{r}_{\text {axle }}\right)$. If, on the other hand, the system radius is entered into the relation, then $D_{m} / r$ is the ratio of the centroid's vertical motion to the upper limit for possible vertical motion $\left(2 \mathrm{r}_{\text {system }}\right)$. The experimental question, then, is: Are judgments of wheel-like motion related to the vertical movement of the centroid relative to its translation ( $\mathrm{r}=$ axle radius) or to its limit of possible vertical motion $(r=$ system radius $)$ ?
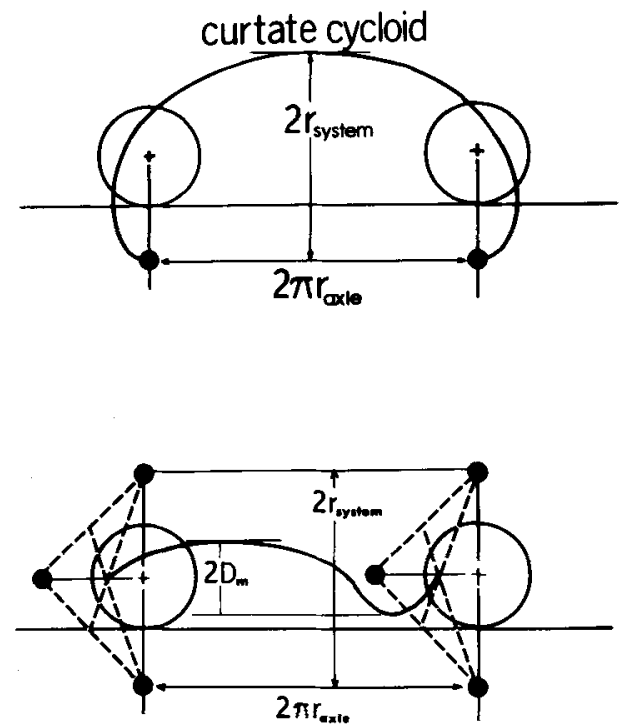

Figure 6. The top panel shows the motion path of a point mounted beyond the perimeter of a rolling wheel. The bottom panel presents the perceived motion path for the centroid of a rolling system of lights, with each light mounted beyond the perimeter of the motion-generating wheel. In both panels, $r_{\text {system }}$ equals the distance of the furthest light from the wheel's center, and $r_{\text {axle }}$ equals the radius of the wheel generating the motion.

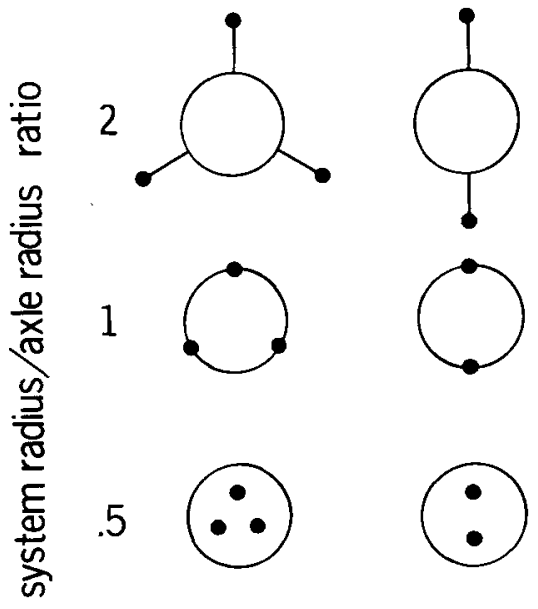

Figure 7. The six stimuli shown in Figure 1 were presented in three manners in Experiment 3. Here, Stimuli 1 and 2 are shown with constant movement generating axles, but with varying system radii.

\section{Method}

Stimuli 1 through 6 of Figure 4 were generated in each of three forms, with a system radius/axle radius ratio of 2.0 , of 1.0 , and of .50 . Stimuli 1 and 2 are shown in these three configurations in Figure 7 . The translational period of the system of lights remains $2 \pi$, if $r$ equals the axle radius, but $\pi, 2 \pi r$, and $4 \pi$ for the three conditions if $r$ equals the system radius. $D_{m} / r$ for all stimuli here are computed for $r$ representing the radius of the system, not the radius of the axle. Ten viewers participated, viewing a single test sequence with all 18 stimulus configurations presented.

\section{Results and Discussion}

As shown in the left panel of Figure $8, D_{m} / r$ ( $\mathrm{r}=$ system radius) continued to account for most of the variance $[F(2,18)=105.7, p<.001]$. We were surprised, however, by the relative ineffectiveness of the proportional differences in horizontal translation. The ratio of the two radii, light system vs. axle, produced no reliable result $[\mathrm{F}(2,18)=3.40$, ns; $\alpha=$ $.05]$. In fact, the effect of the number of lights, always a relatively weak variable in our studies, was more robust than the translational factor $[F(1,9)$ $=6.76, \mathrm{p}<.05]$.

The patterns of results here suggest that judgments of wheel-like movement are related to the vertical excursion of the centroid relative to the limit of possible vertical motion rather than to the translational distance traversed in a period of rotary motion. $\mathrm{D}_{\mathrm{m}} / \mathrm{r}$ holds sway $(\mathrm{r}=-.95, \mathrm{p}<.001)$ if $\mathrm{r}$ is interpreted as the radius of the system. If, on the other hand, $r$ is said to be the radius of the generating axle, the correlation is weaker $(r=-.71)$ precisely because Stimuli 3 through 6 would have indices that vary with translation. That is, in the three conditions, $\mathrm{D}_{\mathrm{m}} / \mathrm{r}$ for Stimuli 3 and 4 , would be $.68, .34$, and .17 and those for Stimuli 5 and 6 would be 1.50 , .75 , and .38 . 
TRANSLATION

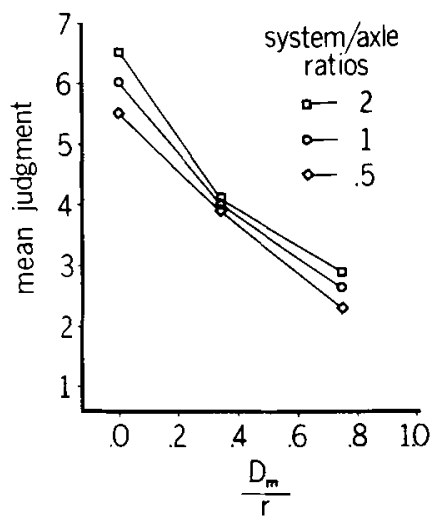

NO TRANSLATION

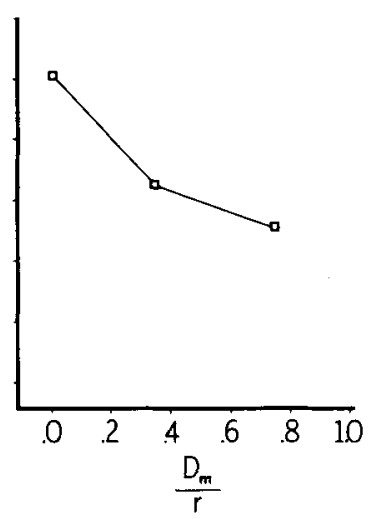

Figure 8. In the lef1 panel, the results of Experiment 3 are shown, with varying degrees of translation as a function of the system/ axle ratios. In the right panel, the results of Experiment 4 are shown for the same stimuli presented without translation.

\section{EXPERIMENT 4: NO HORIZONTAL TRANSLATION}

This experiment provided a strong test of the view that translation is unimportant in the perception of rotary motion by nullifying the translational component altogether.

\section{Method}

Stimuli 1 through 6 were generated so that they rotated once per second, as before, but did so in the middle of the screen without any horizontal translation. Thirteen viewers participated in the study, and were given the same instructions as in the previous studies.

\section{Results and Discussion}

As shown in the right panel of Figure 8, holding the stimuli in the middle of the screen with no translational component did not change the monotonic decreasing relation between judgments of wheellikeness and $\mathrm{D}_{\mathrm{m}} / \mathrm{r}$. The distance measure has a reliable effect $[F(2,24)=25.8, p<.001]$, as does the number of lights ( 2 vs. 3 ) in the system $[F(1,12)$ $=7.0, \mathrm{p}<.05]$. The only difference here is an accentuation of the nonlinear tendency shown in the previous study: Light systems with relatively large $\mathrm{D}_{\mathrm{m}} / \mathrm{r}$ indices tend to be perceived as slightly better than would be predicted from the other studies.

The distance measure predicts wheel-like judgments $(r=-.89, p<.005)$, even with no translational component. The results of this study, like those of the previous one, support the view that the perception of rotary motion is not dependent on the extraction of common vectors moving through space. If this were true, all of these six stimuli should have been perceived as equally wheel-like, since the common vector paths described by every light in every stimulus

is a circle. Instead, it appears that the amount of vertical hopping of the centroid, relative to its limit of possible vertical motion, dictates judgments of wheel-like motion.

\section{EXPERIMENT 5: CIRCULAR TRANSLATION}

In retrospect, it seems reasonable that horizontal translation should not matter much. We can, in fact, see wheels in the real world regardless of whether we follow them with pursuit movement (in which case they stay centered in our visual field much the same as the stimuli in Experiment 4 stayed centered in the middle of the screen), follow them only partially (in which case eyes and wheels move at different speeds, simulated by the conditions of Experiment 3), or follow them not at all (as in the stationary fixation condition of Experiment 2). But in all of these cases the wheel is seen as if it could have been rolling along a flat surface. Our quesiton is: What happens if the path of the wheel is something other than linear and horizontal?

The particular translation pattern we want to consider is circular, one in which a wheel rolls within a ring. This case is of particular interest because Rubin (1927) used this example, where the diameter of the wheel was exactly one-half that of the inner surface of the ring. This arrangement provides a peculiar vector path for the lights on the wheel. Every light on the perimeter of this wheel will move within the outer circle along a straight-line path at the rate of a pendulum (as if viewing a pendulum bob from directly below or directly above). The paths of other lights describe ellipses, and the path of a light in the center, of course, would describe a perfect circle. These relations are shown in the left panel of Figure 9 for Stimulus 4.
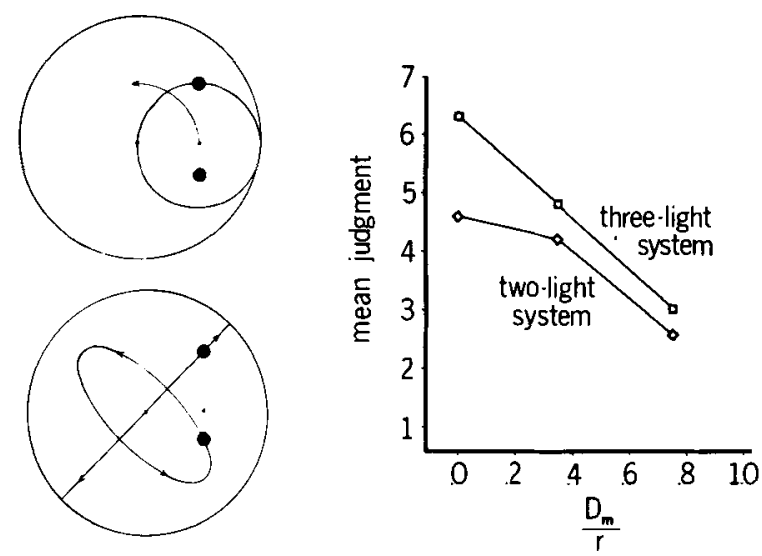

Figure 9. In the left panel, Stimulus 4 is shown as the wheel rolls within a ring twice its diameter, and the straight-line and ellipsoidal vectors are shown. In the right panel, two- and threelight systems are shown separately. 
Rubin's report of the perception of such events is interesting: With two lights mounted opposite one another (like our Stimulus 2), two such flat pendulum movements are seen, and only with six lights is a wheel seen to be rolling within a wheel. In other words, according to Rubin, none of our stimuli should be seen as wheel-like, because the linear vectors of each of the component lights tends to inhibit the perception of a wheel.

\section{Method}

Each of six stimuli was presented as if it were rolling within a ring of twice its diameter. Each made six revolutions as it rolled within the ring three times. Ten viewers rated the movement of the stimuli as to "how much it looked like a wheel rolling within a ring."

\section{Results and Discussion}

The results, shown in the right panel of Figure 9, generally contrast with what Rubin would predict. Our distance measure continued to account for most of the variance $[F(2,18)=27.1, p<.001]$. For the first time in these studies, however, there was a relatively strong effect of the number of lights $[F(1,9)=$ $10.4, p<.02]$. Moreover, there was an interaction of $D_{m} / r$ with the number of lights $[F(2,18)=7.8$, $\mathrm{p}<.05]$. That is, Stimulus 2 the two-light system with a distance measure of .00 ) was seen as less wheellike than would be predicted on the basis of previous data. Thus, Rubin's assertion that two-light systems, undergoing circular translation, are not perceived as potent rotary objects is correct, but not the extent that he would have us believe. The distance measure remains strongly correlated with viewer judgments $(r=-.89, p<.005)$. It must be noted that we suggested the perception of a wheel rolling within a ring to our observers and Rubin did not. This suggestion may account for the difference in our results.

In the discussion of the previous study, we stated that differences in horizontal translatory motion might not matter for the perception of rotary motion, in part because varying amounts of translation are consonant with either pursuit viewing or nonpursuit viewing of rolling wheels. Thus, it is likely that the stimuli of the previous three experiments (and even those of Experiment 1) resemble what we might see every day. However, the type of movement in the present study is found only in the inner workings of a very few mechanical devices, and is seldom or never encountered in everyday life. Yet viewers' judgments can be made with the same facility and exhibit nearly the same regularity of those in previous studies.

\section{EXPERIMENT 6: PROXIMITY CONSTRAINTS ON $\mathbf{D}_{\mathrm{m}} / \mathbf{r}$}

In the first five studies presented here and in the five presented by Proffitt et al. (1979), the predictive strength of our distance measure, $D_{m} / r$, proved remarkably robust against changes in the movements and configurations of the stimuli. Here we attempted to reduce the perceptual utility of the index by devising particular light configurations where the distance index should fail to predict observers' judgments. In particular, we varied the mutual proximity of some of the lights so that two lights would merge into one.

\section{Method}

Ten stimuli were generated, as shown in the left panel of Figure 10. Stimuli $A$ and $H$ are identical to Stimuli 1 and 2 as used in the previous studies. Thus, Stimulus A, for example, has $120^{\circ}$ separating each light. In Stimulus B, we have moved the two lights shown at the bottom towards one another until they differ by only $80^{\circ}$ ( 1.4 radians). Stimuli $\mathrm{C}, \mathrm{D}, \mathrm{E}$, and $\mathrm{F}$ are created in a similar way by reducing that difference to $40^{\circ}, 20^{\circ}, 10^{\circ}$, and $5^{\circ}(.70, .35$, .17 , and .08 radians), respectively. Stimulus $G$ has $0^{\circ}$ separating the two lights, and all that is seen is a brighter light at one end of the system than the other. Thus, Stimuli $G$ and $H$ are identical except for the brightness of one light. Stimuli $I$ and $J$ were added to make more complete the domain of the distance indices for the stimuli, and thus to anchor perceptual judgments. These particular stimuli were used by Proffitt et al. (1979, Experiment 1). The distance index for each stimulus is shown below it. Thirteen viewers participated.

\section{Results and Discussion}

A scatter plot of the 10 stimuli according to their distance measure and judged value is shown in the right panel of Figure 10. Again, $D_{m} / r$ accounted for most of the variance $(r=-.91, p<.001)$. In particular, the linearly decreasing judgments for stimuli of increasing indices failed only for Stimuli E, F, and G. These stimuli had angles separating their closest lights of only $.17, .08$, and .00 radians, and visual angles of only 6,3 , and 0 min of arc. This breakdown of the predictive value of $D_{m} / r$ is thus quite minor, and almost certainly reflects a limit on acuity. The residuals for Stimuli $E, F$, and $G$ between the obtained judgment scores and those predicted from the regression are all within one standard deviation of the residual mean for all points. Moreover, Stimulus $\mathrm{H}$ was judged to be more strongly wheel-like than Stimulus G for 8 of 12 viewers (with one tie), than Stimulus $F$ for 7 of 12 viewers (one tie), and then Stimulus E for 8 of 9 viewers (four ties). Thus, to a remarkable extent, the distance index is unaffected by specific attempts to load stimulus configuration in such a manner as to weaken it.

\section{CONCLUDING COMMENTS}

The major thrust of the present studies is twofold. First, it extends the generality of our previous findings (Proffitt et al., 1979). The distance measure, $D_{m} / r$, suffices as a predictor of observers' judgments of the relative goodness of wheel-like motion for point-light configurations moving in a rotary fashion (a) in rigid and nonrigid systems, (b) with no necessary eye movements, and (c) irrespective of translational fac- 

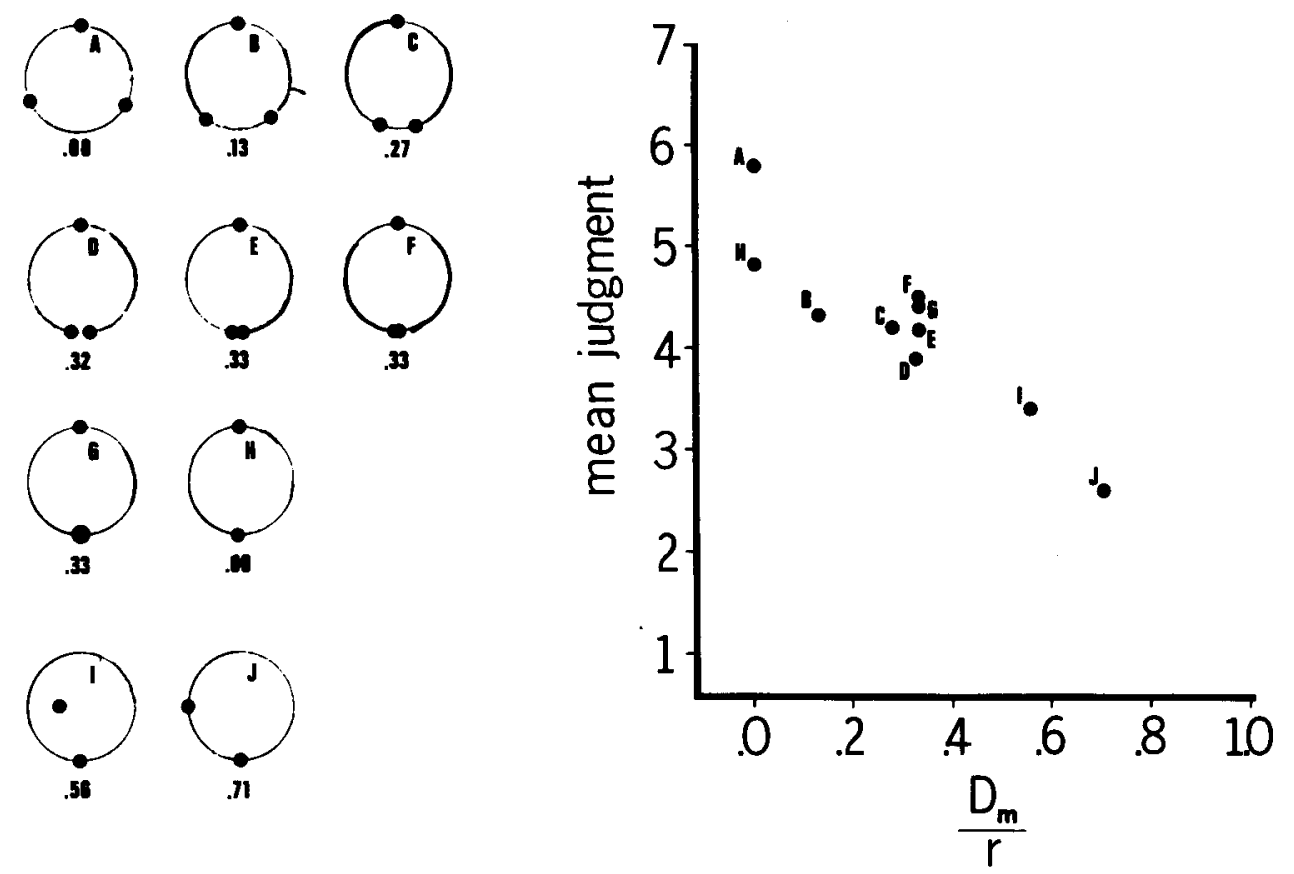

Figure 10. Ten new stimuli are shown, designated $A$ through $J$, with their respective $D_{m} / \mathbf{r}$ indices underneath. In the right panel, their relative judgment scores are displayed.

tors. The predictive value of $\mathrm{D}_{\mathrm{m}} / \mathrm{r}$ is constrained only to a minor degree by the relative separation of the lights as they approach the limits of observer acuity.

Second, the power of prediction that this index affords in the present studies, as well as those of Proffitt et al. (1979), renders more plausible our general view of the perception of moving forms. We contend that the perceptual system selects a mathematical description for a moving form, from among the indefinite number possible, by extracting invariants in a logically ordered, two-step manner. Motion information is, thus, split into two components, one being the internal dynamics of the form and the other being the motion of the form relative to the observer. For lights undergoing wheel-generated motions, the internal dynamics is perceived as the rotational movement of the lights about the centroid of the form defined by taking the lights as vertices. The motion of the form relative to the observer is perceived as the motion path described by the centroid. Our work on the perception of human walkers discussed a conceptually similar abstact center (Cutting, Proffitt, \& Kozlowski, 1978). Using Johansson's point-light techniques, we presented to viewers male and female walkers moving across a display screen. Each walker was represented only as a dynamic system of lights, yet moderately accurate gender recognition was obtained (Barclay, Cutting, \& Kozlowski, 1978; Kozlowski \& Cutting, 1977). Through biomechanical analysis, we were able to locate a point within the torso of the walkers around which all movement occurs. Called the center of moment, this point was relatively higher for females than for males, and viewer performance was highly correlated $(r=.86)$ with its location for each of the different walkers. Moreover, manipulation of this point by use of computergenerated displays yields dynamic arrays systematically judged as male and female walkers (Cutting, 1978).

The Gestalt psychologists argued from such demonstrations as the perception of wheel-generated motions that one does not perceive the motion of a uniform whole by perceiving individually the motions of its parts. We believe this dictum true, yet go further in asserting that the motions of a whole are perceived as the motions of parts. The internal dynamics of a whole are perceived as the movement of parts about a center that is analytic to the whole. The observer-relative motion of the whole is perceived as the dynamics of its center. Although the centers that we have discussed are parts of whole forms, they are not marked by lights at their locations. Just as the physicist determines the centroidcenter of mass-for an object in motion and describes that object's translational dynamics as forces acting, not on the whole, but on that single abstract point, so too the perceptual system appears to select a description for a form in motion by determining the center of its internal dynamics and perceiving translation as the motion path of that center.

\section{REFERENCE NOTE}

1. Verbrugge, R. R., \& Shaw, R. E. How we see events: . A symmetry analysis of perceptual information for rolling objects. Paper presented at the Midwestern Psychological Association, May 1975 . 


\section{REFERENCES}

Barclay, C. D., Cutting, J. E., \& Kozlowski, L. T. Temporal and spatial factors in gait perception that influence gender recognition. Perception \& Psychophysics, 1978, 23, 145-152.

Cutting, J. E. Generation of synthetic male and female walkers through manipulation of a biomechanical invariant. Perception, 1978, 7, 393-405.

Cutting, J. E., Proffitt, D. R., \& Kozlowski, L. T. A biomechanical invariant for gait perception. Journal of Experimental Psychology: Human Perception and Performance, 1978, 4. $357-372$.

DuNCKer. K. Induced motion. In W. D. Ellis (Ed.), A sourcebook of Gestalt psychology. London: Routledge \& Kegan Paul, 1937. (Originally published in German, 1929.)

GiBson, J. J. The perception of the visual world. Boston: Houghton Mifflin, 1950.

Gibson, J. J. The senses considered as perceptual systems. Boston: Houghton Mifflin, 1966.

GiBson, J. J. The ecological approach to visual perception. Boston: Houghton Mifflin, 1979.

Johansson, G. Configuration in event perception. Uppsala, Sweden: Almqvist \& Wiksell, 1950.

Johansson, G. Visual perception of biological motion and a model for its analysis. Perception \& Psychophysics, 1973, 14, 201-211.

Johansson, G. Projective transformations as determining visual space perception. In R. B. MacLeod \& H. L. Pick, Jr. (Eds.), Perception: Essays in honor of James J. Gibson. Ithaca, N.Y: Cornell University Press, 1974. (a)

JoHANSSON, G. Vector analysis in visual perception of rolling motion. Psychologische Forschung, 1974, 36, 311-319. (b)
Johansson, G. Visual motion perception. Scientific American, 1975, 232(6), 76-89.

KoffKa, K. Principles of Gestalt psychology. New York: Harcourt, 1935.

Kozlowski, L. T., \& Cutring, J. E. Recognizing the sex of a walker from a dynamic point-light display. Perception \& Psychophysics, 1977, 21, 575-580.

Proffitt, D. R., Cutting, J. E., \& Stier, D. M. Perception of wheel-generated motions. Journal of Experimental Psychology: Human Perception and Performance, 1979, 5, 289-302.

Rubin, E. Visuell wahrgenommene wirkliche Bewegungen. Zeitschrift fur Psychologie, 1927, 103, 384-392.

Shaw, R. E., \& Pittenger, J. Perceiving the face of change in changing faces: Implications for theory of object perception. In R. E. Shaw \& J. Bransford (Eds.), Perceiving, acting, and knowing. Hillsdale, N.J: Erlbaum, 1977.

STERN, L. O.. \& EMELITY, D. Evidence for frames of reference based on pursuit eye movements. Perception \& Psychophysics, 1978, 24, 521-528.

STOPER, A. E. Apparent motion of stimuli presented stroboscopically during pursuit movement of the eye. Perception \& Psychophysics, 1973, 13, 201-211.

Turvey, M. T. Contrasting orientations to the theory of visual information processing. Psychological Review, 1977, 84, 67-88.

WALLACH, H. Visual perception of motion. In G. Keyes (Ed.), The nature and the art of motion. New York: George Braziller, 1965. (Also in Wallach, H., On perception. New York: Quadrangle, 1976.)

(Received for publication August 28, 1978; revision accepted March 5, 1979.) 